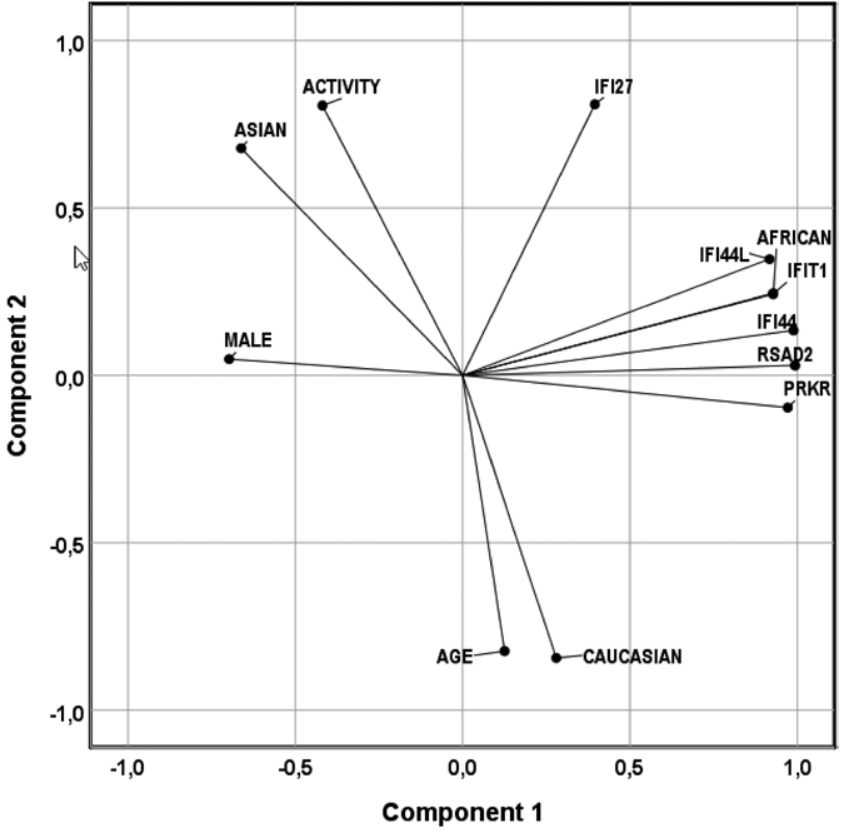

Figure 2. Principal component analysis.

Conclusion: The degree of up-regulation of ISGs in SLE patients shows considerable variation within and between the individual studies. However, a pattern of up-regulation clearly emerges. We find a clustering of 5 prominent genes of the IFN signature (PRKR, RSAD2, IFIT1, IFI44 and IFI44L) and a positive correlation of RSAD2 and PRKR with African ancestry, pointing to the need to take ethnicity into account when using the IFN signature. Our results do not support the use of the IFN signature as traditionally defined as a surrogate marker for disease activity. References:

[1] Kirou, K.A. et. al. Arthritis Rheum. 52, 1491-1503 (2005)

[2] Morand E.F. et. Al. N Engl J Med. (2019)

Table 1. Demographics on the studies in the heatmap. Characteristics refer to SLE patients. HC = healthy controls, SLEDAI = SLE Disease Activity Index, BILAG = British Isles Lupus Assessment Group.

\begin{tabular}{|c|c|c|c|c|c|c|c|c|}
\hline Paper & $\begin{array}{l}\text { SLE } \\
(\mathrm{N})\end{array}$ & $\begin{array}{l}\mathrm{HC} \\
(\mathrm{N})\end{array}$ & $\begin{array}{l}\text { Female } \\
(\mathrm{N})\end{array}$ & $\begin{array}{c}\text { Age } \\
\text { (Mean) }\end{array}$ & $\begin{array}{l}\text { SLEDAI } \\
\text { (Mean) }\end{array}$ & $\begin{array}{l}\text { African } \\
(\mathrm{N})\end{array}$ & $\begin{array}{c}\text { Caucasian } \\
\text { (N) }\end{array}$ & $\begin{array}{c}\text { Asian } \\
\text { (N) }\end{array}$ \\
\hline Panousis, N.I. 2019 & 142 & 58 & 120 & 40 & 5.2 & & 138 & \\
\hline Alcorta, D.A., 2007 & 40 & 28 & 40 & 35 & BILAG: 6.7 & 20 & 18 & \\
\hline Assassi, S., 2010 & 17 & 21 & 16 & 39 & & 2 & 6 & \\
\hline Zhu, H., 2016 & 30 & 25 & 4 & 29 & 12 & & & \\
\hline Li, Q.Z.,2009 & 27 & 11 & 25 & 39 & & 12 & & \\
\hline Chaussabel, D., 2008 & 22 & 12 & 20 & 14 & 12.1 & 6 & 3 & \\
\hline Bouquet, J., 2017 & 11 & 25 & 11 & 51 & & 3 & 5 & 3 \\
\hline Wither, J.E. 2018 & 170 & 22 & 149 & 34 & 8.5 & 40 & 80 & 50 \\
\hline Mackay, M., 2016 & 11 & 20 & 11 & 34 & 13 & & 1 & 4 \\
\hline Yao, Y., 2008 & 41 & 24 & 39 & 40 & & & 41 & \\
\hline Lyons, P.A., 2010 & 13 & 25 & 13 & 47 & & & 12 & 1 \\
\hline Lambers, W.M., 2019 & 39 & 22 & 32 & 43 & $<4$ & & 35 & 1 \\
\hline Baechler, E.C., 2003 & 48 & 42 & & 45 & & & & \\
\hline Han, G.M., 2003 & 10 & 18 & 9 & 29 & 6.1 & & & 10 \\
\hline Ishii, T., 2005 & 31 & 30 & 30 & 34 & 3.8 & & & 31 \\
\hline Tang, J., 2008 & 144 & 60 & 132 & 33 & 6.4 & & & 144 \\
\hline Ye, S., 2003 & 50 & 39 & 45 & 29 & & & & 50 \\
\hline
\end{tabular}

Disclosure of Interests: None declared

DOI: 10.1136/annrheumdis-2020-eular.4784

\section{OP0005 \\ ELEVATED STAT1 EXPRESSION BUT NOT PHOSPHORYLATION IN LUPUS B CELLS CORRELATES WITH DISEASE ACTIVITY AND INCREASED PLASMABLAST SUSCEPTIBILITY}

A. Aue ${ }^{1}$, F. Szelinski ${ }^{1}$, S. Weißenberg ${ }^{1}$, A. Wiedemann ${ }^{1}$, T. Rose ${ }^{1}$, A. Lino ${ }^{1}$, T. Dörner ${ }^{1}$ on behalf of Research Group Prof. Thomas Dörner. ${ }^{1}$ Charité University Hospital, Rheumatology, Berlin, Germany

Background: Systemic lupus erythematosus (SLE) is characterized by two pathogenic key signatures, type I interferon (IFN) (1.) and B-cell abnormalities (2.). How these signatures are interrelated is not known. Type I-II IFN trigger activation of Janus kinase (JAK) - signal transducer and activator of transcription (STAT).
Objectives: JAK-STAT inhibition is an attractive therapeutic possibility for SLE (3.). We assess STAT1 and STAT3 expression and phosphorylation at baseline and after IFN type I and II stimulation in B-cell subpopulations of SLE patients compared to other autoimmune diseases and healthy controls (HD) and related it to disease activity.

Methods: Expression of STAT1, pSTAT1, STAT3 and pSTAT3 in B and T-cells of $21 \mathrm{HD}, 10$ rheumatoid arthritis (RA), 7 primary Sjögren's (pSS) and 22 SLE patients was analyzed by flow cytometry. STAT1 and STAT3 expression and phosphorylation in PBMCs of SLE patients and HD after IFNa and IFNY incubation were further investigated.

Results: SLE patients showed substantially higher STAT1 but not PSTAT1 in $B$ and T-cell subsets. Increased STAT1 expression in B cell subsets correlated significantly with SLEDAI and Siglec-1 on monocytes, a type I IFN marker (4.). STAT1 activation in plasmablasts was IFNa dependent while monocytes exhibited dependence on IFNY.

A

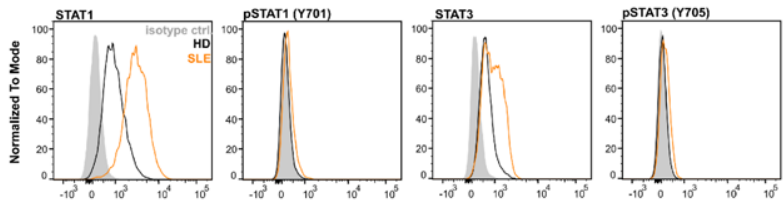

B

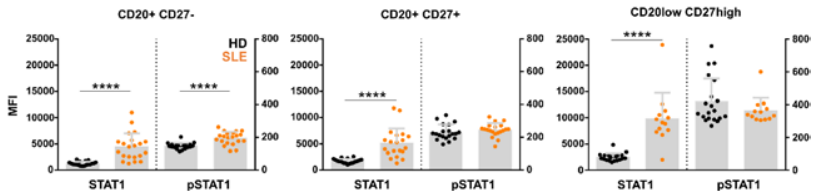

C

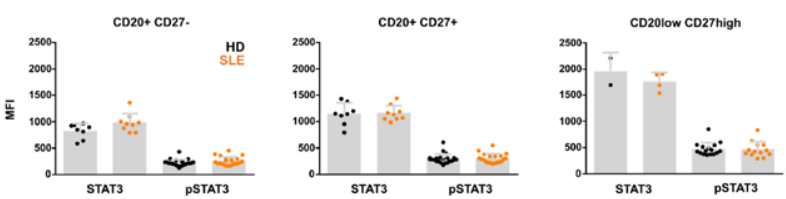

Figure 1. Significantly increased expression of STAT1 by SLE B cells(A) Representative histograms of baseline expression of STAT1, pSTAT1, STAT3 and pSTAT3 in CD19+ B cells of SLE patients (orange), HD (black) and isotype controls (grey). (B) Baseline expression of STAT1 and pSTAT1 or (C) STAT3 and pSTAT3 in CD20+CD27-, CD20+CD27+ and CD20lowCD27high B-lineage cells from SLE (orange) patients compared to those from HD (black). Mann Whitney test; ${ }^{* \star \star \star} p \leq 0.0001$.

A

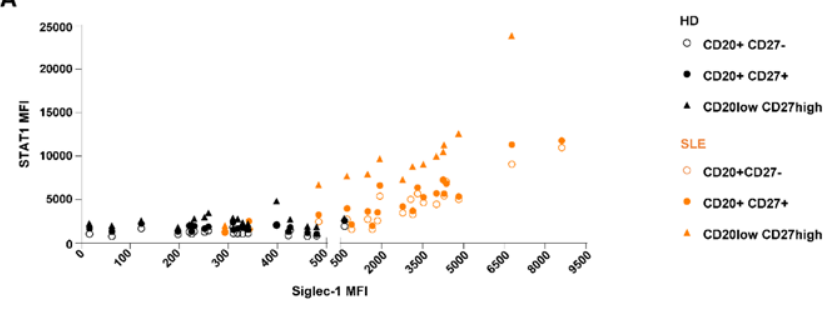

B
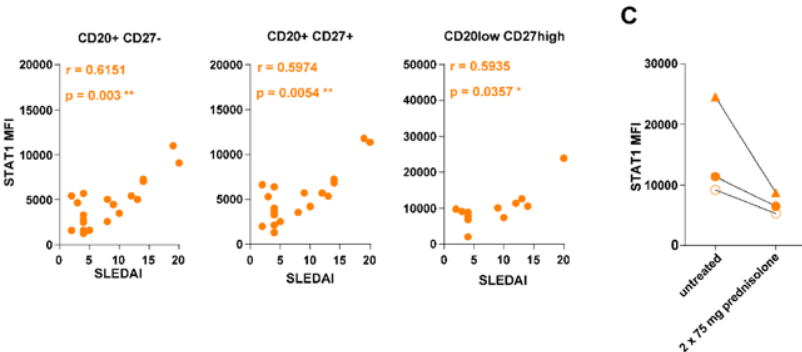

Figure 2. Correlation of STAT1 expression by SLE B cells correlates with type I IFN signature (Siglec-1, CD169) and clinical activity (SLEDAI).Correlation of STAT1 expression in CD20+CD27- näive $(p<0.0001, r=0.8766), C D 20+C D 27+$ memory $(p<0.0001, r=0.8556)$ and CD20lowCD27high $(\mathrm{p}<0.0001, \mathrm{r}=0.9396)$ B cells from SLE patients with (A) Siglec-1 (CD169) expression on CD14+ cells as parameter of type I IFN signature and (B) lupus disease activity (SLEDAI score). Spearman rank coefficient $(r)$ was calculated to identify correlations between these parameters. ${ }^{*} p \leq 0.05,{ }^{\star *} p \leq 0.01$. (C) STAT1 expression in B cell subsets of a previously undiagnosed, active SLE patient who was subsequently treated with two dosages of prednisolone and reanalyzed. 
Conclusion: Enhanced expression of STAT1 by B-cells candidates as key node of two immunopathogenic signatures (type I IFN and B-cells) related to important immunopathogenic pathways and lupus activity. We show that STAT1 is activated upon IFNa exposure in SLE plasmablasts. Thus, Jak inhibitors, targeting JAKSTAT pathways, hold promise to block STAT1 expression and control plasmablast induction in SLE.

References:

[1] Baechler EC, Batliwalla FM, Karypis G, Gaffney PM, Ortmann WA, Espe KJ, et al. Interferon-inducible gene expression signature in peripheral blood cells of patients with severe lupus. Proc Natl Acad Sci U S A. 2003;100(5):2610-5.

[2] Lino AC, Dorner T, Bar-Or A, Fillatreau S. Cytokine-producing B cells: a translational view on their roles in human and mouse autoimmune diseases. Immunol Rev. 2016;269(1):130-44.

[3] Dorner T, Lipsky PE. Beyond pan-B-cell-directed therapy - new avenues and insights into the pathogenesis of SLE. Nat Rev Rheumatol. 2016;12(11):645-57.

[4] Biesen R, Demir C, Barkhudarova F, Grun JR, Steinbrich-Zollner M, Backhaus $\mathrm{M}$, et al. Sialic acid-binding Ig-like lectin 1 expression in inflammatory and resident monocytes is a potential biomarker for monitoring disease activity and success of therapy in systemic lupus erythematosus. Arthritis Rheum. 2008;58(4):1136-45.

Disclosure of Interests: Arman Aue: None declared, Franziska Szelinski: None declared, Sarah Weißenberg: None declared, Annika Wiedemann: None declared, Thomas Rose: None declared, Andreia Lino: None declared, Thomas Dörner Grant/research support from: Janssen, Novartis, Roche, UCB, Consultant of: Abbvie, Celgene, Eli Lilly, Roche, Janssen, EMD, Speakers bureau: Eli Lilly, Roche, Samsung, Janssen

DOI: 10.1136/annrheumdis-2020-eular.2955

\section{Metabolic pathways during the regulation of inflam- mation and immunity}

\section{OP0006 \\ ABNORMAL IRON METABOLISM AND MITOCHONDRIAL DYSFUNCTION: INVESTIGATING A NOVEL PATHOLOGICAL MECHANISM IN SYSTEMIC LUPUS ERYTHEMATOSUS}

C. Wincup ${ }^{1}$, G. Robinson ${ }^{1}$, T. Mcdonnell ${ }^{1}$, A. Radziszewska ${ }^{1}$, F. Farinha ${ }^{1}$, A. Rahman ${ }^{1} .{ }^{1}$ University College London, Department of Rheumatology, London, United Kingdom

Background: Iron is vital for numerous essential physiological processes including erythropoiesis and energy metabolism (as iron is found in the mitochondrial electron transport chain, the central site of ATP production). Iron homeostasis is tightly controlled by a number of regulators including; 1 . Hepcidin, which prevents iron release from stores (under the influence of IL6 and IL1 $\beta$ ); 2 . Ferritin, an iron storage protein; 3. Lipocalin-2 (LCN2), which is released upon innate immune activation that induces iron sequestration; 4 . Transferrin, which binds circulating iron and enables its transport to effector cell targets; 5 . Haptoglobin, which binds free haemoglobin and assisting iron recycling; 6. Erythropoietin (EPO), which stimulates erythropoiesis as a result of hypoxia.

Chronic inflammation may result in dysregulation of iron metabolism and in turn impair mitochondrial function yet little is known regarding how these processes change in systemic lupus erythematosus (SLE).

Objectives: In this study, we investigated how dysregulation of iron metabolism may occur in SLE and subsequently sought to identify how a lack of iron may ultimately induce abnormal mitochondrial function.

Methods: 1. Investigating abnormal iron metabolism in SLE. Serum samples from patients with SLE $(n=39)$ and healthy controls $(H C, n=17)$ were assessed hepcidin, IL-1ß, IL-6, ferritin, LCN2, EPO, haptoglobin and transferrin levels by ELISA. Hierarchical cluster analysis of normalised data (converted to Z-scores) was performed using MeV software in order to characterise patient groups based upon iron metabolism profile. Anti-dsDNA antibody titres, complement C3 levels and SLEDAI-2K were excluded to limit the influence of these variables on cluster analysis. Results were presented as a heatmap.

2. Studying mitochondrial function in iron deficiency and SLE. Peripheral blood mononuclear cells (PBMCs) from HCs and patients with SLE were analysed using Seahorse Respirometry, which measures mitochondrial oxygen consumption rate (a measure of energy metabolism dependent upon oxidative phosphorylation). To assess differences between health, iron deficiency and SLE 3 groups were assessed; 1. PBMCs derived from $\mathrm{HCs}$;2. PBMCs from patients with SLE; 3 . Healthy PBMCs cultured in iron deficient condition, in which cells were treated with the potent iron chelator, Deferiprone.

Results: Figure 1a demonstrates that four groups were identified following cluster analysis. In spite of excluding markers of disease activity, these groups showed significant differences in SLEDAI-2K (shown in Figure 1b). In summary, patients with more active disease (Groups $C$ and D) showed higher levels of hepcidin (which prevents the release of iron from stores, under the influence of $\mathrm{IL}-1 B$ and $\mathrm{IL}-6)$ and reduced transferrin thus suggesting that iron is inefficiently transported when compared with those with less active disease (in Groups A and B).
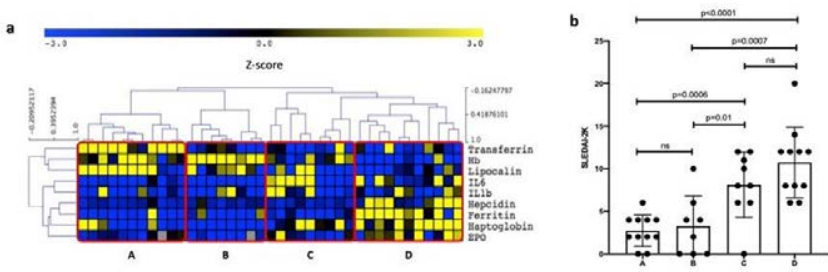

Figure 1a: Results of hierarchical clustering identified four patient groups (A-D) based upon key regulators of iro metabolism

figure 1b: Differences in disease activity (SLEDAI-2K) between each group produced from hierarchical cluster analysis

Figure $2 \mathrm{a}$ demonstrates that basal mitochondrial respiration is significantly reduced in PBMCs derived from healthy controls when grown in iron deficiency conditions (following treatment with Deferiprone and is lower still in those with SLE. Figure $2 \mathrm{~b}$ shows that PBMCs from patients with SLE have reduced maximal mitochondrial respiration capacity that is comparable to the levels seen in iron deficient healthy PBMCs.

\section{Figure 2}
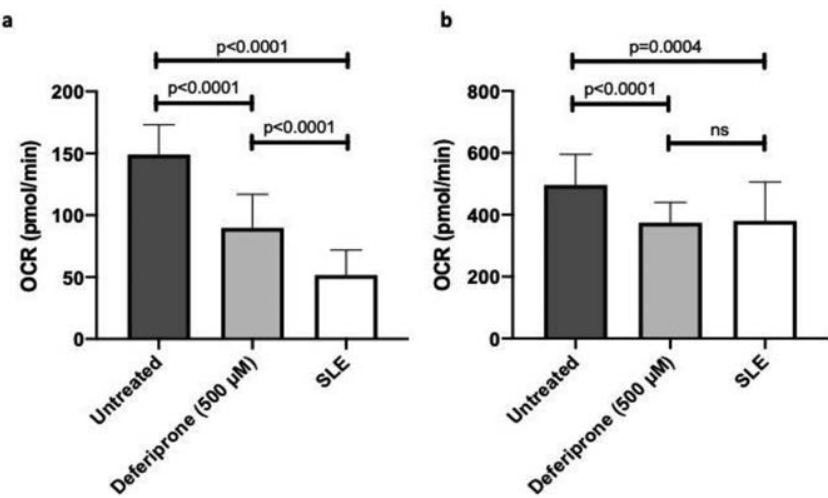

Conclusion: Patients with SLE demonstrate abnormalities in iron metabolism that results in cellular iron deficiency as iron is not released from stores, nor adequately transported at the rate required to meet physiological demands. Furthermore, PBMCs derived from patients with SLE who impaired basal and maximal respiration that is comparable with healthy PBMCs treated potent iron chelation. This suggests that abnormal iron metabolism may in turn limit mitochondrial energy metabolism in SLE and represents a potential future therapeutic target

References: Nil

Acknowledgments: Versus Arthritis (Grant No 594143) and LUPUS UK

Disclosure of Interests: None declared

DOI: 10.1136/annrheumdis-2020-eular.2487

\section{OP0007-PARE CAMPAIGN TO PROMOTE PHYSICAL ACTIVITY \& EXERCISE TO RMD PATIENTS THROUGH EDUCATION AND PRACTICE}

\section{A. lacovou ${ }^{1} .{ }^{1}$ Cyprus League Against Rheumatism, Nicosia, Cyprus}

Background: Eular give's a lot of attention to outline the need of a change in RMD patients life style that is very well outlined into the 2018 Eular recommendations for Physical Activity (PA).

Objectives: Driven by those recommendations that says that "PA should be an integral part of standard care throughout the course of disease," CypLAR decided to create a campaign to promote PA through educating RMD patients on the PA benefits, make them to change their life style and enroll them to PA programs. More over we want to inform Rheumatologist and HPR's on that effort and enroll them to that campaign.The CypLAR' $s$ goal through that campaign is to manage and enroll as much as possible patients to PA Programs for a continual period of about 10 months. 